\title{
Correlaciones fenotípicas entre características de importancia económica en ovinos Suffolk Down y Merino Precoz en la zona central de Chile
}

\author{
Phenotypic correlations among economic important traits in Suffolk Down and Merino Precoz \\ sheep in central Chile
}

\author{
F Lembeye $^{\mathrm{a} *}$, G Castellaro ${ }^{\mathrm{b}}$, JC Magofke ${ }^{\mathrm{b}}$, H Uribe $^{\mathrm{b}}$ \\ ${ }^{a}$ Institute of Veterinary, Animal and Biomedical Sciences, Massey University, New Zealand. \\ bDepartamento de Producción Animal, Facultad de Ciencias Agronómicas, Universidad de Chile, Santiago, Chile.
}

\begin{abstract}
SUMMARY
Suffolk Down (S) and Merino Precoz (MP) are the main sheep breeds reared in central and south central area of Chile. In both breeds the main source of income for farmers is weaned lamb sale, therefore traits associated to reproduction, live weight and growth were included in the selection objectives of genetic improvement programs. In dual purpose breeds, fleece weight, and length and diameter of the fiber were also included in genetic programs. The objective of this study was to estimate phenotypic correlations among some economically important traits in meat and dual purpose sheep. The traits studied in S and MP were birth weight (BW) and birth to weaning growth rate (GR). For MP breed, greasy fleece weight (GFW), fiber diameter (FD) and staple length (SL) were also included. Variance components estimation was done by least squared means. Estimated variances for BW in $\mathrm{S}$ and MP were: $0.58 \mathrm{~kg}^{2}$ and $0.52 \mathrm{~kg}^{2}$, respectively. Regarding GR, in the same order, estimated variances were $1,150 \mathrm{~g}^{2}$ and $1,208.7 \mathrm{~g}^{2}$. Further, in MP were also estimated the variances for GFW $\left(0.296 \mathrm{~kg}^{2}\right)$, FD $\left(3.31 \mu \mathrm{m}^{2}\right.$ and SL $\left(293.8 \mathrm{~mm}^{2}\right)$. Estimated phenotypic correlation between BW and GR for $\mathrm{S}$ lambs was $0.12 \pm 0.05,(\mathrm{P}=0.036)$. For MP breed the correlation between these traits was $-0.05 \pm 0.04(\mathrm{P}=0.14)$. In MP breed, only phenotypic correlation between GFW and SL was significant. The estimated correlations were: GFW-DF $(0.043 \pm 0.07)$, GFW-SL $(-0.207 \pm 0.10)$ and DF-SL $(0,035 \pm 0.10)$. Among wool and live weight traits, estimated phenotypic correlations were: GFW-BW $(0.02 \pm 0.07)$, FD-BW $(-0.08 \pm 0.10)$, SL-BW $(0.12 \pm 0.10)$, GFW-GR $(-0.11 \pm 0.07)$, FD-GR $(-0.03 \pm 0.10)$ and SL-GR $(0.05 \pm 0.10)$. Neither of this values was statistically different from zero (P > $0.05)$. It si concluded that there exist a large environmental variability for live weight and growth.
\end{abstract}

Key words: phenotypic correlations, sheep, suffolk, merino.

\section{RESUMEN}

Suffolk Down (S) y Merino Precoz (MP) son las principales razas utilizadas en la zona centro y centro sur de Chile, siendo la principal fuente de ingreso la venta de corderos al destete por lo que características ligadas a la eficiencia reproductiva y peso vivo y crecimiento debieran ser parte de los objetivos de selección en programas de mejoramiento genético. En razas doble propósito, peso del vellón, largo y finura también debieran ser consideradas en programas genéticos. El objetivo del estudio fue estimar correlaciones fenotípicas para algunas características de interés económico en ovinos de carne y doble propósito. En ambas las razas se estudió el peso al nacimiento (PN) y tasa de crecimiento nacimiento-destete (TC). En MP se consideró el peso del vellón sucio (PVS), diámetro de fibra (DF) y largo de mecha (LM). La estimación de varianzas se realizó mediante mínimos cuadrados. Las varianzas fenotípicas estimadas para PN en S y MP fueron: $0,58 \mathrm{~kg}^{2}$ y $0,52 \mathrm{~kg}^{2}$, respectivamente, y en el mismo orden para TC: $1.150 \mathrm{~g}^{2}$ y $1.208,7 \mathrm{~g}^{2}$. Para características de lana en MP las varianzas fueron: $0,296 \mathrm{~kg}^{2}$ para PVS, $3,31 \mu \mathrm{m}^{2}$ para DF y $293,8 \mathrm{~mm}^{2}$ para LM. La correlación fenotípica estimada entre PN y TC en S fue 0,12 $\pm 0,05$ ( $\mathrm{P}=0,036)$. En MP el valor de la correlación entre PN y TC fue $-0,05 \pm 0,04$ ( $\mathrm{P}=0,14)$, mientras que las correlaciones fenotípicas entre DF, PVS y LM no difieren estadísticamente de cero (P > 0,05), a excepción de PVS-LM. Los valores estimados fueron: PVS-DF $(0,043 \pm 0,07)$ PVS-LM $(-0,207 \pm$ $0,10)$ y DF-LM $(0,035-0,10)$. Las correlaciones fenotípicas entre características de vellón con peso vivo y crecimiento tampoco difieren estadísticamente de cero (P > 0,05), los valores estimados fueron: PVS-PN $(0,02 \pm 0,07)$, DF-PN $(-0,08 \pm 0,10)$, LM-PN $(0,12 \pm 0,10)$, PVS-TC $(-0,11 \pm 0,07)$, DF-TC $(-0,03 \pm$ $0,10)$ y LM-TC $(0,05 \pm 0,10)$. Se concluye que existe una gran variación observada, de naturaleza ambiental, para características de peso vivo y crecimiento.

Palabras clave: correlaciones fenotípicas, ovinos, Suffolk, merino.

\section{INTRODUCCIÓN}

En el rubro ovino existen distintas características de interés económico dependiendo de la aptitud de la raza.

Aceptado: 29.08.2013.

* Private Bag 11,222, Massey, New Zealand; felipelembeye@gmail.com
El ovino Suffolk Down (carne) y Merino Precoz (carne y lana) son las principales razas utilizadas por ganaderos de la zona centro y centro sur de Chile. Tanto en la razas de carne como doble propósito, la principal fuente de ingreso es a través de la venta de corderos al destete. Por consiguiente, características ligadas a la eficiencia reproductiva y también de peso vivo y crecimiento debieran ser parte de los objetivos de selección en programas de mejoramiento genético. 
En ovinos doble propósito como el Merino Precoz, distintos autores señalan que características como peso del vellón, largo y finura debieran ser considerados en programas de mejoramiento genético a través de selección (Mueller 1985, Whiteley 1994, Atkins 1997). En Chile, se han hecho pocos estudios que describan la asociación fenotípica que existe entre estas características de interés para ser empleados en programas de selección.

El objetivo de este estudio es estimar correlaciones fenotípicas para algunas características de interés económico en ovinos de carne y doble propósito, manejados en condiciones de secano en la zona central de Chile.

\section{MATERIAL Y MÉTODOS}

\section{CARACTERÍSTICAS GENERALES DEL ÁREA DEL ESTUDIO}

Los datos utilizados en esta investigación pertenecen a los rebaños Suffolk Down y Merino Precoz de la Estación Experimental Rinconada de Maipú de la Universidad de Chile (3331' Lat. Sur; $70^{\circ} 50^{\prime}$ Long. Oeste; 470 m.s.n.m.) (García y col 1993). El clima es mediterráneo semiárido, con un régimen térmico que se caracteriza por temperaturas que oscilan entre una máxima en enero de $28,2^{\circ} \mathrm{C}$ y una mínima en julio de $4,4^{\circ} \mathrm{C}$. El período libre de heladas es de 231 días, con un promedio de 11 heladas por año (Santibáñez y Uribe 1993). Los registros pluviométricos de la Estación Experimental reportan un monto anual promedio de $305 \mathrm{~mm}$ (período 1958-2011), éste se concentra en un $94,4 \%$ entre los meses de abril y septiembre.

El sistema de explotación del rebaño es de carácter extensivo, con una parición al año y el recurso alimenticio es exclusivamente el pastizal natural. En la Estación Experimental de Rinconada se realiza un encaste por medio de monta natural a partir de mediados de enero hasta mediados de marzo. Las pariciones comienzan a mediados de junio y se prolongan hasta mediados de agosto. Los corderos son vendidos al destete, generalmente durante el mes septiembre y hasta principios del mes de octubre.

\section{ESTIMACIÓN DE PARÁMETROS FENOTÍPICOS}

Las características consideradas en este estudio para las razas Suffolk Down (S) y Merino Precoz (MP) fueron peso al nacimiento $(\mathrm{PN})$ y tasa de crecimiento nacimientodestete (TC). Adicionalmente en la raza MP se consideraron características de vellón: peso del vellón sucio (PVS), diámetro de fibra (DF) y largo de mecha (LM), todas ellas medidas solamente en hembras a la primera esquila. El DF y LM fueron medidos mediante análisis OFDA 2000® (Analizador Óptico del Diámetro de Fibra), en muestras de lana obtenidas de la zona media del costillar de las hembras dos semanas antes de la esquila. Para las características anteriormente mencionadas se estimaron varianzas $\left(\sigma_{P}^{2}\right)$ y correlaciones $\left(r_{P_{X Y}}\right)$ fenotípicas.
La estimación se realizó mediante un análisis de varianza de mínimos cuadrados, según el método descrito por Harvey (1990), utilizando el programa computacional MINITAB (2006) (Minitab 15 Statistical software. Minitab Inc., Pennsylvania State University, PA, USA).

No se consideraron corderos con un peso al nacimiento inferior a 2,5 kilos; pesos al destete inferiores a 25 kilos, como tampoco una edad al destete inferior a 60 días. En el caso de ovinos S de un total de 563 registros se utilizaron en el análisis de la información relacionada con PN y TC: 219 y 111 registros para corderos nacidos únicos y mellizos, respectivamente. Para la raza MP, en el mismo orden el número de registros fueron: 466 y 249, esto de un total de 1.419 registros totales.

Para características de vellón, los registros disponibles para PVS fueron: 127 y 66 para borregas nacidas únicas y mellizas. Para DF y LM, 81 y 25 en el mismo orden.

Los efectos fijos incluidos en los modelos preliminares para PN fueron: año de nacimiento; edad de la oveja, agrupada en cinco clases; estación de parto; tipo de parto; sexo y todas las interacciones dobles entre los efectos mencionadas anteriormente. El modelo estadístico que define lo anterior fue el siguiente:

$Y_{i j k l m n}=\mu+A_{i}+E_{j}+E P_{k}+T_{l}+S_{m}+($ Int.dobles $)+e_{i j k l m n}$

Donde $Y_{i j k l m n}=$ representa el dato productivo de PN. $\mu=$ promedio general. $A_{i}=$ efecto del i-ésimo año (i $=2008$, 2010 y 2011). $E_{j}=$ efecto de la j-ésima edad de la oveja en años $(\mathrm{j}=2, \ldots, 6) . E P_{k}=$ efecto de la k-ésima estación de parto $(\mathrm{k}=$ temprano, tardío; definiéndose época temprana como el período en que ocurrieron el $50 \%$ de los primeros partos.). $T_{l}=$ efecto del l-ésimo tipo de parto (l = único, doble). $S_{m}=$ efecto del m-ésimo sexo del cordero $(\mathrm{m}=$ hembra, macho $)$. (Int.dobles $)=$ interacciones dobles entre las fuentes de variación. $e_{i j k l m n}=$ residual no explicado por el modelo $\sim N\left(0, \sigma_{e}^{2}\right)$.

En el caso de la TC se consideran los mismos efectos fijos utilizados en el análisis de PN, pero se incluyó además como covariable la edad al destete $\left(\beta_{X_{i}-\bar{X}}\right)$.

En el análisis de PVS, el modelo utilizado fue el siguiente:

$Y_{i j k l}=\mu+A_{i}+E_{j}+T_{k}+\beta_{\left(X_{i}-\bar{X}\right)}+$ (Int. dobles $)+e_{i j k l}$

Donde los elementos son los descritos anteriormente excepto donde $\mathrm{i}=2010$ y 2011, la j-ésima edad de la madre fue agrupada en tres clases (2-3, 4-5 y 6 años), esto porque análisis previos mostraron diferencias no significativas entre las subclases que fueron reagrupadas en las nuevas clases, y $\left(\beta_{X_{i}-\bar{X}}\right)$ es la covariable de la edad a la esquila.

Para el análisis del DF y LM el modelo incluyó solamente los efectos de la edad de la madre (pero agrupada 
únicamente en dos categorías: 2 y 3 o más años) y el tipo de parto (únicos y mellizos). No se consideró el efecto del año ya que los registros disponibles para estas variables correspondieron únicamente al año 2010. En estas variables el modelo estadístico se redujo a:

$$
Y_{i j k}=\mu+E_{i}+T_{j}+\beta_{\left(X_{i}-\bar{X}\right)}+(\text { Int. dobles })+e_{i j k}
$$

Donde $Y_{i j k}=$ representa un dato productivo (DF ó LM), $\left(\beta_{X_{i}-\bar{X}}\right)=$ efecto fijo de la edad a la esquila y los otros elementos de acuerdo a lo descrito anteriormente.

Los modelos finales, conservaron solamente los efectos que resultaron estadísticamente significativos $(\mathrm{P}<0,05)$. Las varianzas fenotípicas $\left(\sigma_{P}^{2}\right)$ se estimaron a través del cuadrado medio del error (CME) que resultó de cada uno de los modelos descritos anteriormente (Harvey 1990).

La varianza y desviación estándar $\left(\sigma_{\mathrm{p}}\right)$ no son comparables entre diferentes características con distinta unidad de medida. Se calculó por consiguiente el coeficiente de variación (C.V.); el C.V. expresa la desviación estándar como porcentaje de la media fenotípica, mostrando una mejor interpretación porcentual del grado de variabilidad que la desviación típica o estándar y permite también comparar la variación de distintas variables.

C.V. $=\frac{\sigma_{P}}{\mu} \cdot 100$

Donde $C . V$. = coeficiente de variación. $\sigma_{\mathrm{p}}=$ desviación estándar. $\mu=$ promedio población para la característica $i$. Estimación de correlaciones fenotípicas

La correlación fenotípica entre dos características $\left(r_{P_{X Y}}\right)$ fue estimada, con los datos corregidos por todas aquellas fuentes de variación significativas en ambas características, a través de la siguiente fórmula (Cameron, 1997):

$r_{P_{X Y}}=\frac{\operatorname{Cov}_{P_{X Y}}}{\left(\sigma_{X}\right)\left(\sigma_{Y}\right)}$

Donde $r_{\mathrm{P}_{\mathrm{XY}}}$ y $\operatorname{Cov}_{\mathrm{P}_{\mathrm{XY}}}$ son la correlación y covarianza fenotípica, respectivamente, entre dos características, $\sigma_{i}$ es la desviación estándar fenotípica de la i-ésima característica.

Para corregir los datos crudos, se calcularon factores de ajuste aditivos para cada uno de los efectos ambientales. Para ello, se calculó el desvío entre el promedio mínimo cuadrado de cada efecto significativo, con el promedio general para la característica de acuerdo a la siguiente metodología: (adaptado de Fernández y col 2010).

$\widehat{P}_{l}=P_{i}-\left(\bar{X}_{l}-\overline{\bar{X}}_{l}\right)$

Donde: $\widehat{P}_{i}=$ valor fenotípico corregido de la característica i. $P_{i}=$ valor fenotípico sin corregir de la característica $i . \overline{X_{i}}=$ promedio mínimo cuadrático de cada clase de la característica $i . \overline{X_{i}}=$ promedio general de la característica $i$.

\section{RESULTADOS}

Las fuentes de variación que se informan en los análisis de varianza consideran sólo los efectos que resultaron estadísticamente significativos $(\mathrm{P}<0,05)$. Los resultados para ambas razas de PN y TC se muestran en los cuadros 1 y 2 , respectivamente.

\section{PESO AL NACER}

Los efectos de año, tipo de parto y sexo fueron significativos en explicar la variabilidad de peso al nacimiento en los corderos de ambas razas. El efecto de la edad de la madre sólo fue importante en el S. En el MP, la edad de la madre fue significativa solo al 10\%, aún así se consideró este efecto como una fuente de variación también importante en el MP, ya que el nivel de significancia resultó muy próximo al 5\% $(\mathrm{P}=0,055)$. Además, para esta última raza también fue importante el efecto estación de parto. Por otra parte, las interacciones no resultaron importante en ambas razas $(\mathrm{P}>0,05)$. Los promedios generales para PN en S y MP fueron: 4,42 y 4,29 kg, respectivamente. Con esta información se calculó el C.V. para ambas razas. Se registraron similares C.V. para PN en ambas razas, con valores de 17,3 y $16,8 \%$, para el S y $\mathrm{MP}$, respectivamente. Las varianzas fenotípicas para $\mathrm{PN}$, en las razas S y MP, fueron 0,58 y $0,52 \mathrm{kilos}^{2}$, respectivamente (cuadro 1).

\section{TASA DE CRECIMIENTO}

En contraste a lo encontrado para PN, los corderos MP tuvieron un mayor incremento de peso nacimientodestete que los corderos $\mathrm{S}$ en las tres temporadas estudiadas. El promedio general para esta variable fue: $278,3 \mathrm{y}$ 298,8 g día $^{-1}$, respectivamente para ovinos S y MP. Los C.V. para esta variable fueron inferiores a los obtenidos para PN. En ambas razas la variación observada fue similar, siendo 12,2 y 11,6\% los C.V. para las razas S y MP, respectivamente. Por otra parte, las varianzas fenotípicas para tasa de crecimiento en las razas S y MP fueron 1.150 y $1.208 \mathrm{~g}^{2}$ por día, respectivamente (cuadro 2).

\section{CARACTERÍSTICAS DE VELLÓN}

Los resultados de los análisis de varianza para estas características realizados con datos de borregas de primera esquila se presentan en el cuadro 3.

El C.V. registrado para PVS fue $14,0 \%(\mu=3,89 \mathrm{~kg})$. De las características de vellón estudiadas en este trabajo, el diámetro de fibra presentó el menor coeficiente de variación $(7,7 \%)(\mu=23,75 \mu \mathrm{m})$, mientras que la mayor variación se encontró para LM cuyo valor fue 18,4\% ( $\mu$ = 93,34 mm). Las varianzas fenotípicas para peso del vellón, diámetro de fibra y largo de mecha fueron $0,296 \mathrm{~kg}^{2}$, $3,31 \mu \mathrm{m}^{2}$ y $293,8 \mathrm{~mm}^{2}$, respectivamente (cuadro 3). 
F LEMBEYE Y COL

Cuadro 1. Análisis de varianza para peso al nacer en corderos Suffolk y Merino Precoz.

Birth weight analysis of variance for Suffolk and Merino lambs

\begin{tabular}{lcccccc}
\hline & \multicolumn{5}{c}{ Raza del cordero } \\
\cline { 2 - 7 } \multicolumn{1}{c}{ Fuente de variación } & \multicolumn{5}{c}{ Suffolk Down } & Merino Precoz \\
\cline { 2 - 7 } & GL & CM & Valor P & GL & CM & Valor P \\
\hline Año & 2 & 14,5 & $<0,01$ & 2 & 21,9 & $<0,01$ \\
Edad de la madre & 4 & 2,7 & $<0,01$ & 4 & 1,20 & 0,055 \\
Tipo de parto & 1 & 68,1 & $<0,01$ & 1 & 110,9 & $<0,01$ \\
Sexo & 1 & 5,7 & $<0,01$ & 1 & 4,3 & $<0,01$ \\
Estación de parto & - & - & N.S. & 1 & 2,01 & 0,049 \\
Error & 321 & 0,58 & & 705 & 0,52 & \\
\hline Total & 329 & & & 714 & & \\
\hline
\end{tabular}

GL: grados de libertad. CM: cuadrado medio. Valor P: valor de probabilidad

Cuadro 2. Análisis de varianza para tasa de crecimiento en corderos Suffolk y Merino Precoz.

Growth rate analysis of variance for Suffolk and Merino lambs.

\begin{tabular}{lcccccc}
\hline \multirow{2}{*}{ Fuente de variación } & \multicolumn{3}{c}{ Suffolk Down } & \multicolumn{3}{c}{ Merino Precoz } \\
\cline { 2 - 6 } & GL & CM & Valor P & GL & CM & Valor P \\
\hline Año & 2 & 7.921 & $<0,01$ & 2 & $12.884,3$ & $<0,01$ \\
Edad de la madre & 4 & 3.526 & 0,017 & 4 & $83.093,2$ & $<0,01$ \\
Tipo de parto & 1 & 88.847 & $<0,01$ & 1 & $122.732,0$ & $<0,01$ \\
Sexo & 1 & 7.027 & 0,014 & - & - & N.S. \\
Año*Edad de la madre & - & - & N.S. & 8 & $39.203,8$ & $<0,01$ \\
Año*Tipo de parto & - & - & N.S. & 2 & $49.049,0$ & $<0,01$ \\
Estación de parto*Sexo & - & - & N.S. & 3 & $76.493,7$ & $<0,01$ \\
Edad al destete & 1 & 122.149 & $<0,01$ & 1 & $409.506,3$ & $<0,01$ \\
Error & 324 & 1.150 & & 693 & $1.208,7$ & \\
\hline Total & 329 & & & 714 & & \\
\hline
\end{tabular}

GL: grados de libertad. CM: cuadrado medio. Valor P: valor de probabilidad.

Cuadro 3. Análisis de varianza para peso del vellón sucio, diámetro de fibra y largo de mecha en borregas Merino Precoz.

Analysis of variance for greasy fleece weight and, fibre diameter and staple length in Merino ewe.

\begin{tabular}{|c|c|c|c|c|c|c|c|c|c|}
\hline \multirow{2}{*}{ Fuente de variación } & \multicolumn{3}{|c|}{ Peso del Vellón (kg) } & \multicolumn{3}{|c|}{ Diámetro de fibra $(\mu \mathrm{m})$} & \multicolumn{3}{|c|}{ Largo de mecha (mm) } \\
\hline & GL & $\mathrm{CM}$ & Valor P & GL & $\mathrm{CM}$ & Valor P & GL & $\mathrm{CM}$ & Valor P \\
\hline Edad esquila & 1 & 1,4908 & 0,026 & - & - & - & - & - & - \\
\hline Edad de la madre & 2 & 1,0198 & 0,034 & 1 & 13,175 & 0,049 & 1 & 0,03 & 0,857 \\
\hline Tipo de parto & 1 & 4,2644 & $<0,01$ & 1 & 7,73 & 0,129 & 1 & 1,87 & 0,175 \\
\hline Error & 188 & 0,296 & & 102 & 3,31 & & 102 & 293,8 & \\
\hline Total & 192 & & & 105 & & & 105 & & \\
\hline
\end{tabular}

GL: grados de libertad. CM: cuadrado medio. EdM: edad de la madre. TP: tipo de parto. Valor P: valor de probabilidad al 5\%. 


\section{CORRELACIONES FENOTÍPICAS ENTRE PN Y TC}

La correlación estimada en corderos $\mathrm{S}$ resultó positiva aunque baja, siendo su valor de 0,12 $\pm 0,05$ ( $\mathrm{P}=$ 0,036). En el MP el valor de la correlación, entre PN y $\mathrm{TC}$, fue $-0,05 \pm 0,04$, valor que no resultó significativo $(\mathrm{P}=0,14)$.

\section{CORRELACIONES FENOTÍPICAS ENTRE CARACTERÍSTICAS DE LA LANA}

En la raza MP se calcularon correlaciones fenotípicas entre PVS, DF y LM cuyos resultados se presentan en el cuadro 4. Las correlaciones fenotípicas calculadas entre DF con PVS y con LM no difieren estadísticamente de cero $(\mathrm{P}>0,05)$ siendo estos resultados no consistentes con la literatura revisada. La única correlación significancia estadística fue entre PVS con LM, no obstante, su significancia estadística ( $\mathrm{P}=0,03)$, el valor difiere sustancialmente a la revisión de Safari y col (2005) y a la de otros investigadores.

\section{CORRELACIONES FENOTÍPICAS ENTRE CARACTERÍSTICAS DEL VELLÓN Y CRECIMIENTO EN OVINOS MERINO PRECOZ}

Las correlaciones entre características de vellón con PN y TC, en la raza MP, se presentan en el Cuadro 5. De acuerdo a los resultados ninguna correlación fenotípica entre características de vellón con PN y TC fue significativa $(\mathrm{P}>0,05)$.

Cuadro 4. Correlaciones fenotípicas entre peso vellón sucio (PVS), diámetro de fibra (DF) y largo de mecha (LM) en ovinos Merino Precoz.

Phenotypic correlations among greasy fleece weight and, fibre diameter and staple length in Merino Precoz

\begin{tabular}{ccc}
\hline PVS-DF & PVS-LM & DF-LM \\
\hline $0,043 \pm 0,07$ & $-0,207 \pm 0,10$ & $0,035 \pm 0,10$ \\
$($ Valor $\mathrm{P}=0,664)$ & $($ Valor $\mathrm{P}=0,03)$ & $($ Valor $\mathrm{P}=0,721)$ \\
\hline
\end{tabular}

\section{DISCUSIÓN}

Las variaciones encontradas para PN son similares a la revisión de literatura realizada por Fogarty (1995) en razas de carne y doble propósito (17\%). Safari y col (2005) en cambio, informan una mayor variación para peso al nacimiento en razas de carne $(19,2 \pm 1,0 \%)$ en comparación a las de doble aptitud (16,5 $\pm 0,6 \%)$. En cambio, para TC los valores son inferiores (12,2 en $\mathrm{S}$ y $11,6 \%$ en MP) a los informados por Safari y col (2005) quienes producto de 19 estimaciones, sin distinción de razas, obtuvieron un C.V. promedio de 19,8 $\pm 1,5 \%$. La mayor variación obtenida por estos autores coincide con las publicaciones de Rao y Notter (2000) quienes informan un C.V. de $23 \%$ en ovinos S, y con las de Notter y Hough (1997); Bromley y col (2000) y Rao y Notter (2000) que obtuvieron un C.V. que fluctúo entre 15 y $20 \%$ en distintas razas.

El C.V. encontrado para PVS se encuentra en el rango inferior de lo obtenido por otros autores. Bromley y col (2000) y Rao y Notter (2000) informan un C.V. para PVS entre 15 y $22 \%$. Safari y col (2005) obtienen un C.V. promedio para razas de doble propósito de 16,2 $\pm 0,6 \%$. Para DF en cambio, valor es muy similar al C.V. promedio informado por Safari y col (2005) en razas de doble propósito $(7,2 \pm 0,5 \%)$, y de lana $(7,4 \pm 0,3 \%)$. Esta variación es ligeramente inferior a la reportada previamente por Fogarty (1995), quién informa un C.V. de 8\% para esta DF únicamente en razas de lana. La variación obtenida en LM $(18,4 \%)$ es ligeramente menor al publicado por Notter y col (2007); sin embargo, es sustancialmente mayor al informado por Bromley y col (2000) quienes consideraron distintas razas de doble propósito obteniendo un promedio de $13 \%$. El valor calculado también es mayor al de la revisión de literatura de Safari y col (2005) donde los autores obtienen un C.V. de 14,0 $\pm 1,0 \%$.

En relación a la correlación fenotípica entre $\mathrm{PN}$ con TC, para la raza $\mathrm{S}\left(r_{P}=0,12\right)$, este resultado es similar a las estimaciones de Mousa y col (1999) y Roshanfekr y col (2011), quienes obtienen una correlación fenotípica de 0,13 en ovinos compuestos y 0,12 en ovinos árabes. Por otra parte, y en relación al MP $\left(r_{P}=-0,05\right)$, Dixit y col (2001) y Ozder y col (2009) encontraron una correlación fenotípica baja y similar a la estimada en el presente

Cuadro 5. Correlaciones fenotípicas entre características de vellón con peso al nacer (PN) y tasa de crecimiento pre-destete (TC) en ovinos Merino Precoz.

Phenotypic correlations among fleece and growth traits

\begin{tabular}{cccccc}
\hline PVS-PN & DF-PN & LM-PN & PVS-TC & DF-TC & LM-TC \\
\hline $0,02 \pm 0,07$ & $-0,08 \pm 0,10$ & $0,12 \pm 0,10$ & $-0,11 \pm 0,07$ & $-0,03 \pm 0,10$ & $0,05 \pm 0,10$ \\
$(\mathrm{P}=0,78)$ & $(\mathrm{P}=0,41)$ & $(\mathrm{P}=0,21)$ & $(\mathrm{P}=0,14)$ & $(\mathrm{P}=0,77)$ & $(\mathrm{P}=0,61)$ \\
\hline
\end{tabular}


trabajo $\left(r_{P}=-0,01\right.$ y 0,03 en ovinos de la raza Merino y Bharat, respectivamente). Las estimaciones de este trabajo, sin embargo, son inferiores a los valores publicados por Thrift y col $(1973)\left(r_{P}=0,28\right)$ en ovinos Suffolk, Maria y col (1999) $\left(r_{P}=0,37\right)$ en la raza Romanov y Jurado y col (1994) $\left(r_{P}=0,35 \pm 0,53\right)$ en Merino Español. Los resultados sugieren que no hay una clara relación entre PN y la velocidad de crecimiento post natal en el MP, ya que los incrementos de peso registrados para ambas razas, no ocurrieron en las mismas etapas del año. Incluso, el mayor incremento de peso se observó en el año 2011, temporada en la cual se obtuvo el menor peso al nacimiento en el MP. Aún así estos resultados concuerdan con Olson y col (1976), quienes indican que las correlaciones obtenidas en condiciones de pastoreo extensivo y en suelos marginales, son generalmente bajas, incluso pueden ser nulas, esto como producto de una alta participación del ambiente. Sin embargo, esto no implica que haya independencia genética (Cardellino y Rovira 1987).

Como se señaló en la sección anterior, los valores de correlaciones fenotípicas encontradas en este trabajo no son consistentes con la literatura extranjera. De acuerdo a la revisión de Safari y col (2005), las correlaciones fenotípicas de DF con PVS y LM son ampliamente superiores a las encontradas en este trabajo, los valores que informan fueron: $0,31(0,14-0,45)$ para DF con PVS producto de 15 estimaciones, y $0,19(0,01-0,37)$ para DF con LM. Correlaciones fenotípicas inferiores a la revisión realizada por Safari y col (2005) entre PVS-DF, pero superiores a las encontradas en este trabajo, son informadas por Mueller y col (2003) en dos planteles Merino en la Patagonia Argentina $\left(r_{P}=0,1\right.$ y 0,$\left.25 ; \mathrm{P}<0,05\right)$. Otros autores que han estimado correlaciones fenotípicas entre DF con LM en ovinos Merino son: Atkins (1997) $\left(r_{P}=\right.$ 0,15); Mortimer y Atkins (1989) $\left(r_{P}=0,09\right)$, Swan y col (1995) $\left(r_{P}=0,16\right)$, Greeff y col (1995) $\left(r_{P}=0,11-0,26\right)$, Cloete y col (1998) $\left(r_{P}=0,13 \pm 0,02\right)$. A excepción de los resultados de Mortimer y Atkins (1989), la literatura indica correlaciones fenotípicas superiores a 0,10 entre estas dos variables. En contraste con lo anterior, la correlación fenotípica entre PVS con LM estimada en este trabajo resulto estadísticamente significativa $(P=0,03)$, no obstante, este valor difiere sustancialmente al valor de $0,32(0,18-0,45)$ de la revisión de Safari y col (2005) y trabajos anteriores (Swan y col 1995: $r_{P}=0,37$, Mortimer y Atkins 1989: $\left.r_{P}=0,28 \pm 0,04\right)$.

Para características de vellón con crecimiento, los resultados de este trabajo no concordaron con aquellos presentados en la revisión de literatura de Safari y col (2005) donde las correlaciones fenotípicas entre PN con PVS y DF son: $0,24(0,05-0,41)$ y $-0,05(-0,11 ;-0,01)$, respectivamente. No se encontró una correlación fenotípica significativa entre PN y LM en la literatura revisada en esta investigación.

Dado las inconsistencias en los valores de correlaciones fenotípicas entre características de lana y entre estas con PN y TC en MP, se sugiere que el número de regis- tros de vellón, disponibles en este trabajo, puede no ser suficientes para una mejor estimación de estos parámetros fenotípicos. Por otra parte, según Safari y col (2005) las correlaciones fenotípicas entre TC con características de la lana son positivas, pero bajas: $0,25(0,11-0,39) ; 0,05$ $(-0,06$ a 0,16$)$ y $0,01(-0,03$ a 0,05$)$ para TC con PVS, DF y LM, respectivamente.

De acuerdo a los resultados obtenidos en este trabajo, para ovinos de carne y doble propósito existe una gran variación observada que es de naturaleza ambiental para características de peso vivo y crecimiento; lo anterior se corrobora con los bajas correlaciones obtenidas entre PN y TC para ambas razas, y que son consistente además con otros trabajos realizados en ovinos en condiciones extensivas.

Para características de vellón, sin embargo, dado el bajo número de registros disponibles, los resultados contrastan de manera importante a la de otros autores, por tanto, mayores antecedentes se necesitan para estimar, con mayor precisión, la asociación entre estas variables para ovinos MP en condiciones de pastoreo extensivo en pastizales de secano semiárido.

\section{REFERENCIAS}

Atkins K. 1997. Genetic improvement of wool production. In: Piper L, Ruvinsky A (eds). The genetics of sheep. CAB International, Pp 471-504.

Bower K. 2000. Analysis of Variance (ANOVA) Using MINITAB. Scientific Computing \& Instrumentation 17, 64-65.

Bromley C, G Snowder, L Van Vleck. 2000. Genetic parameters among weight, prolificacy, and wool traits of Columbia, Polypay, Rambouillet, and Targhee sheep. J Anim Sci 78, 846-858.

Cameron N. 1997. Selection Indices and Prediction of Genetic Merit in Animal Breeding. CAB International, Wallingford, UK.

Cardellino R, J Rovira. 1987. Mejoramiento genético animal. $1^{\text {ra }}$ ed. Editorial Agropecuaria Hemisferio Sur, Montevideo, Uruguay.

Cloete S, J Olivier, M Snyman, E Du Toit. 1998. Genetic parameters and trends in a selection experiment for increased clean fleece weight involving South African Merinos. Aus $J$ Exp Agric 38, 427-432.

Dixit S, J Dhilon, D Sing. 2001. Genetic and nongenetic parameter estimates for growth traits of Bharat Merino lambs. Small Rumin Res 42, 101-104.

Fernández G, B Mernies, C Silviera. 2010. Guía teórico- práctica de Mejora Genética. Curso de Mejora Genética Área VII. Oficina de Publicaciones de la Facultad de Veterinaria, Universidad de la República, Montevideo, Uruguay.

Fogarty N. 1995. Genetic parameters for live weight, fat and muscle measurements, wool production and reproduction in sheep: a review. Anim Breed Abst 63, 101-143.

García G. 1986. Características de las razas ovinas criadas en Chile. En: García G (ed). Producción ovina. Universidad de Chile, Facultad de Ciencias Agropecuarias y Forestales, Santiago, Chile, Pp 9-21.

García G, S Díaz, J Saini, J Rojas, M Recalde, H Soto. 1993. Un sistema semi intensivo de producción para ovinos en 
el secano interior de la zona central. Simiente 63, 137-145.

Greeff J, R Lewer, R Ponzoni, I Purvis. 1995. Staple strength: progress towards elucidating its place in Merino breeding. Proc. Australian Association. J Anim Breed Gen 11, 595601.

Harvey W. 1990. User's guide for LSMLMW and MIXMDL. PC-2 version. Mixed Model Least-Squares and Maximum Likelihood Computer Program. Columbus, Ohio, USA.

Jurado J, A Alonso, R Alenda. 1994. Selection response for growth in a Spanish Merino flock. J Anim Sci 72, 14331440 .

Maria G, K Boldman, L Van Vleck. 1993. Estimates of variances due to direct and maternal effects for growth traits of Romanov sheep. J Anim Sci 71, 845-849.

Mortimer S, K Atkins. 1989. Genetic evaluation of production traits between and within flocks of Merino sheep. I. Hoggets fleece weight, body weight and wool quality. Aust J Agric Res 40, 433-443.

Mousa E, L Van Vleck, K Leymaster. 1999. Genetic parameters for growth traits for a composite terminal sire breed of sheep. J Anim Sci 77, 1659-1665.

Mueller J. 1985. Implementación de planes de mejoramiento ovino. I Objetivos de mejoramiento y criterios de selección. INTA EEA Bariloche Comunicación Técnica PA6.

Mueller JP, F Bidinost, HR Taddeo. 2003. Parámetros genéticos en dos planteles Merino de la Patagonia. Revista de Investigaciones Agropecuarias 32, 161-172.

Notter D, J Hough. 1997. Genetic parameter estimates for growth and fleece characteristics in Targhee sheep. $J$. Anim Sci 75, 1729-1737.

Notter D, L Kuehn, R Kott. 2007. Genetic analysis of fiber characteristics in adult Targhee ewes and their relationship to breeding value estimates derived from yearling fleeces. Small Rumin Res 67, 164-172.

Olson L, G Dickerson, H Glimp.1976. Selection criteria for intensive market lamb production: Growth traits. J Anim Sci 43, 78-89.

Ozder M, T Sezenler, A Refik, A Ceyhan. 2009. Genetic and non-genetic parameter estimates for growth traits in Turkish Merino lambs. J Anim Vet Adv 8, 1729-1734.

Rao S, D Notter. 2000. Genetic analysis of litter size in Targhee, Suffolk, and Polypay sheep. J Anim Sci 78, 2113-2120.

Roshanfekr H, M Mamouei, K Mohammadi, E Rahmatnejad. 2011. Estimatin of genetic and environmental parameters affected pre-weaning traits of Arabic lambs. J Anim Vet Advan 10, 1239-1243.

Safari E, N Fogarty, A Gilmour. 2005. A review of genetic parameter estimates for wool, growth, meat and reproduction traits in sheep. Livest Prod Sci 92, 271-289.

Santibáñez Q, M Uribe. 1993. Atlas Agroclimático de Chile. Regiones sexta, séptima, octava y novena. Laboratorio de Agroclimatología. Departamento de Ingeniería y Suelos. Universidad de Chile, Facultad de Ciencias Agrarias y Forestales, Santiago.

Swan A, J Lax, I Purvis. 1995. Genetic variation in objectively measured wool traits in CSIRO's fine wool flock. Proc. Aust Assoc Anim Breed Genet 11, 516-520.

Thrift F, J Whiteman, D Kratzer. 1973. Genetic analysis of preweaning and postweaning lamb growth traits. J Anim Sci 36, 640-643.

Whiteley K. 1994. The influence of wool fiber characteristics on processing and garment performance. In: Azzarini M, Cardellino R (ed). IV World Merino Conference, Montevideo Uruguay, Pp 209-227. 
